\title{
Neuroimaging correlates of handwriting quality as children learn to read and write
}

\section{Paul Gimenez ${ }^{1}$, Nicolle Bugescu ${ }^{1,2}$, Jessica M. Black ${ }^{3}$, Roeland Hancock ${ }^{1}$, Kenneth Pugh ${ }^{4}$, Masanori Nagamine ${ }^{1,5}$, Emily Kutner ${ }^{1,2}$, Paul Mazaika ${ }^{6}$, Robert Hendren ${ }^{1}$, Bruce D. McCandliss ${ }^{7}$ and Fumiko Hoeft ${ }^{1,4,6,8 *}$}

1 Division of Child and Adolescent Psychiatry, Department of Psychiatry, UCSF, San Francisco, CA, USA

2 Department of Psychology, Pacific Graduate School of Psychology, Palo Alto University, Palo Alto, CA, USA

${ }^{3}$ Graduate School of Social Work, Boston College, Chestnut Hill, MA, USA

${ }^{4}$ Haskins Laboratories, Yale University, New Haven, CT, USA

${ }^{5}$ Division of Behavioral Sciences, National Defense Medical College Research Institute, Tokorozawa, Saitama, Japan

${ }^{6}$ Department of Psychiatry and Behavioral Sciences, Center for Interdisciplinary Brain Sciences Research, Stanford University School of Medicine, Stanford, CA, USA

7 Department of Psychology and Human Development, Vanderbilt University, Nashville, TN, USA

${ }^{8}$ Department of Neuropsychiatry, Keio University School of Medicine, Shinjuku-ku, Tokyo, Japan

\section{Edited by:}

Pierluigi Zoccolotti, Sapienza University of Rome, Italy

\section{Reviewed by:}

Scott K. Holland, Cincinnati Children's Research Foundation, USA

Donatella Spinelli, Università di Roma "Foro Italico," Italy

\section{${ }^{*}$ Correspondence}

Fumiko Hoeft, Division of Child and Adolescent Psychiatry, Department of Psychiatry, University of California, 401 Parnassus Ave. Box 0984-F, San Francisco, CA 94143 , USA

e-mail: fumiko.hoeft@ucsf.edu
Reading and writing are related but separable processes that are crucial skills to possess in modern society. The neurobiological basis of reading acquisition and development, which critically depends on phonological processing, and to a lesser degree, beginning writing as it relates to letter perception, are increasingly being understood. Yet direct relationships between writing and reading development, in particular, with phonological processing is not well understood. The main goal of the current preliminary study was to examine individual differences in neurofunctional and neuroanatomical patterns associated with handwriting in beginning writers/readers. In 46 5-6 year-old beginning readers/writers, ratings of handwriting quality, were rank-ordered from best to worst and correlated with brain activation patterns during a phonological task using functional MRI, and with regional gray matter volume from structural T1 MRI. Results showed that better handwriting was associated negatively with activation and positively with gray matter volume in an overlapping region of the pars triangularis of right inferior frontal gyrus. This region, in particular in the left hemisphere in adults and more bilaterally in young children, is known to be important for decoding, phonological processing, and subvocal rehearsal. We interpret the dissociation in the directionality of the association in functional activation and morphometric properties in the right inferior frontal gyrus in terms of neural efficiency, and suggest future studies that interrogate the relationship between the neural mechanisms underlying reading and writing development.

Keywords: phonological processing, voxel-based morphometry, functional MRI, inferior frontal gyrus pars triangularis, writing, reading

\section{INTRODUCTION}

Writing by hand occupies 30-60\% of a child's school day (Stewart, 1992; Simner, 1998; Feder and Majnemer, 2007; Sassoon, 2007) and correlates with self-esteem and future academic success. Children with deficient handwriting (10-30\% of children; Karlsdottir and Stefansson, 2002) take longer to complete writing tasks such as homework, which can increase the difficulty of schoolwork and result in oppositional attitudes toward writing assignments that can generate problems both at school and at home (Racine et al., 2008). Crucially, handwriting performance also shares links with other language related skills. Of particular relevance, there are important associations between reading and learning to write. Studies have shown that learning to write can improve letter perception (Longcamp et al., 2005), pseudoletter learning (Richards et al., 2011), and word reading (e.g., Berninger et al., 2004, 2006a; James and Engelhardt, 2012).
Correspondingly, children with learning disabilities such as developmental dyslexia, a specific reading impairment that is believed to have phonological deficits at its core, often display writing difficulties (O'Hare and Khalid, 2002).

With the increasing integration of computers into the education system, the implied implications of reduced handwriting practice have strengthened the interest of scientific investigators. Recent neuroimaging studies have concluded that while freeform handwriting practice clearly supports reading acquisition, typing (Longcamp et al., 2005) and even tracing (James and Engelhardt, 2012) do not. Impressively, James and Engelhardt (2012) showed that preliterate children recruit well established reading related brain regions, such as the fusiform gyrus, posterior parietal cortex, and the inferior frontal gyrus, during letter processing exclusively after handwriting practice compared to typing or tracing. The emerging consensus is that the motor 
experience of manually creating letterforms helps children discriminate the essential properties of each letter, which leads to more accurate representations bolstering both skilled letter recognition and later reading fluency. Therefore, understanding the underlying neurological mechanisms that support handwriting development is important not only for its independent relevance to educational achievement, but also for its supportive role in successful acquisition of other language skills such as reading.

The neurological basis underlying handwriting is not well understood but converging evidence points to key regions including: (a) the fusiform gyrus, which has apparent selectivity to letter (James and Gauthier, 2006) and word stimuli (Cohen et al., 2000; Cohen and Dehaene, 2004) over other visual stimuli and may provide a perceptual component for deriving "word-form" representations that facilitate grapheme writing (Dehaene et al., 2005; James, 2010); (b) the superior parietal lobule (SPL), a region important for carrying out actions in space (Goodale and Milner, 2005) that is thought to be involved in both visuospatial and visuomotor processing (Petrides and Pandya, 1984; Morecraft et al., 2004; Segal and Petrides, 2012), and the execution of writing sequences (Otsuki et al., 1999); (c) the inferior frontal gyrus (IFG), implicated for its involvement in phonological processes (Eckert et al., 2003) and its associations with encoding letterforms and words (Grafton et al., 1997; Berninger and Winn, 2006b; Longcamp et al., 2008); and (d) Exner's area, thought to be the interface of orthographic or graphemic representations and the complex movement sequences necessary for generating letters and words (Anderson et al., 1990; Lubrano et al., 2004; Roux et al., 2009) and may also be involved in retrieving letter shapes from memory (James and Gauthier, 2006).

While advances have been made, a complete understanding of the brain's writing system remains elusive. The inherent complexities involved in the task of writing, coupled with the excessive variability of its definition in the existing literature, make it challenging to delineate the extent of neuronal specialization specific to handwriting from other inter-related aspects, such as spelling. In a recent neuroimaging metaanalysis of writing in adults, however, authors dissociated linguistic input and motoric aspects of writing and identified IFG for processing linguistic input as it relates to writing, and left superior frontal sulcus/middle frontal gyrus (Exner's), left superior parietal lobule, and the right cerebellum as "writing-specific" regions (Planton et al., 2013). Another study has shown that the brain differentially recruits specialized regions based on a multiplicity of letter representations (e.g., motoric similarities "B" vs. "P," visual similarities "A" vs. "R," abstract similarities "A" vs. "a”) (Rothlein and Rapp, 2014). What is lacking is detailed examination of the emergence of "neural specialization" during the period when writing skills develop and the brain basis of the underlying process (except see work by Karen James cited in this paper). Further, more investigations of association between the brain basis of writing and other processes of written language such as reading is greatly needed. Findings from such studies may not only offer important insights to improve research methodology and educational instruction, but may also contribute to a fuller understanding of the development of written language processing in the human brain.
The present study sought to focus on the neural correlates of handwriting quality in children at the beginning of formal handwriting instruction. Operationally, handwriting quality refers to the legibility, form, slant, spacing, and general appearance of letters and words. Handwriting researchers have generally agreed on the relevance of these key features (Freeman, 1959; Kaminsky and Powers, 1981; Graham, 1982; Ziviani and Elkins, 1984; Graham and Weintraub, 1996). Given that handwriting exposure in preliterate children has been associated with reading related processes such as letter perception and related brain activation (James and Engelhardt, 2012), it is plausible to consider that especially during early stages of development, handwriting also share links with phonological processing a skill that for decades has been casually linked to reading acquisition (Castles and Coltheart, 2004; Byrne et al., 2008). Therefore, we sought to investigate the unproven idea that handwriting and reading may rely on a common neuroanatomical mechanism at an early developmental stage of reading/writing. We therefore hypothesized handwriting quality will be associated with neuroanatomical patterns in one or more of the following: (a) IFG if phonological decoding coding is relevant to handwriting quality, (b) Exner's area if successful integration of orthography and motor programs are relevant to handwriting quality, (c) SPL if sequential motor movements and/or kinesthetic modulation are relevant to handwriting quality, and/or (d) fusiform gyrus if visual letter or word recognition is relevant to handwriting skill. Then, in order to investigate the direct relationship of handwriting and reading abilities, in particular of phonological processing, we associated handwriting quality with brain activation during a task aimed at engaging the brain's phonological processing circuit. If handwriting is associated with the development of reading, and phonological processing plays a causal and reciprocal relationship with reading acquisition, we hypothesized that brain activation patterns associated with phonological processing, may also be associated with handwriting skills in these emergent readers / writers.

\section{MATERIALS AND METHODS}

Our data come from a study focused on examining brain activation during phonological processing and the relationship between reading-related behavioral measures. While the original study was not focused on the brain basis of handwriting, and hence the behavioral measures and fMRI tasks were not necessarily optimized for the purpose of the current study, yet these data provided an opportunity to investigate whether neuroanatomical patterns and brain activation during phonological processing are associated with handwriting in beginning readers and writers.

\section{PARTICIPANTS}

A total of 51 (29 boys, 22 girls) healthy, native English-speaking 5 - and 6-year-old children (aged $5.59 \pm 0.42$ ) toward the beginning of formal schooling participated in this study. Standard behavioral assessments of the children, along with MRI data were collected. We later excluded five left-handed children, leaving 46 remaining right-handed children to be included in all analyses unless there was missing data or excessive movement motion or severe scanner artifacts (fMRI analyses, $N=41$ ). While we did not exclude children based on attention deficit hyperactivity 
disorder (ADHD) for example, the children in this study did not have any parental report of formal diagnosis of neurological or psychiatric disorders besides specific learning disabilities; they were not on medication and had no contraindications to MRI. Behavioral Assessment System for Children -2 (Reynolds and Kamphaus, 2006) showed that all children were within typical range.

To help prepare participants for imaging, parents received a packet of informational material, including a CD of common scanner sounds and a DVD of a child going into the scanner. Parents were instructed to review these supplemental materials with their children to familiarize and desensitize participants to the scanner environment. In addition, children participated in a guided MRI simulation at the center where they practiced lying still in the bore and underwent training to minimize motion related artifacts. Participants with excessive, uncorrectable motion were eliminated from the study.

The Stanford University Panel on Human Subjects in Medical Research and the University of California, San Francisco Human Research Protection Program approved the study and informed consent and assent were obtained from parents/guardians and participants, respectively.

\section{BEHAVIORAL MEASURES}

We administered a standard battery of neuropsychological assessments, which included the Woodcock-Johnson III (WJ-III) Spelling (Woodcock et al., 2001), an untimed real-word spelling test, in order to assess spelling accuracy and handwriting quality (see below); the Beery Visual-Motor Integration (BVMI; Beery and Beery, 2004), where children copied and traced a series of moderately complex geometric figures; and the Oromotor Sequences subtest from the Developmental Neuropsychological Assessment (NEPSY-II; Korkman et al., 2007) to assess oralmotor praxis, or the ability to sequence oral-motor movements without articulation difficulty, without visual demands. Additionally, the Home Literacy Inventory (Marvin and Ogden, 2002) was used to investigate the differences in the exposure and practice of reading activities at home.

\section{HANDWRITING QUALITY}

In order ensure participants were unaware that their handwriting was under investigation, handwriting samples were drawn from the WJ-III Spelling subtest were used as a basis for assessing and defining handwriting skills. Two blinded investigators, who were trained to score handwriting quality holistically based on letterform, slant, spacing and general appearance irrespective of spelling errors and speed, each rank-ordered ( $1=$ poor handwriting, $51=$ best handwriting) participants' writing sample from best to worst three times. Since spelling inaccuracies can inadvertently bias rankings, writing samples included both letters and small words. Intraclass correlation coefficients were calculated to examine intra-rater reliability (Cronbach's alpha $=0.994$ for rater 1; 0.989 for rater 2), and inter-rater reliability (Cronbach's alpha $=0.980$ ) was calculated after the three sets of scores were averaged across raters. The final ranking used was based on the mean of each investigator's scores.

\section{VISUOMOTOR (COPYING) SKILLS}

A subset of test items (items 17-19) from the BMVI task was selected by the investigators to evaluate visuomotor skills; these items were developmentally appropriate, yet were also sufficiently difficult. Specifically, these were the most difficult items (non-letter objects) that all participants were able to complete. According to the manual, the validity and reliability of the task are sufficient for the age of our participants (Beery, 1997). Following the same rank-ordering procedures as for handwriting quality, two blind investigators rated participants' reproductions, which were based on copying geometric shapes $(1=$ poor reproduction, 51 = best reproduction). Intraclass correlation coefficients were calculated to examine intra-rater reliability (Cronbach's alpha $=$ 0.993 for rater $1 ; 0.974$ for rater 2) and inter-rater reliability (Cronbach's alpha $=0.969$ ) was calculated after the three sets of scores were averaged across raters. The final ranking used was based on the mean of each investigator's scores.

\section{FUNCTIONAL MRI TASKS}

Three tasks measuring a range of cognitive abilities were used to investigate neurological associations to handwriting. The first was a phonological processing task in which participants were asked to determine if the first sound of the names of two pictures of common objects matched (Figure 1A). This task was adapted from a sound-matching subtask of the Comprehensive Test of Phonological Processing (Wagner et al., 1999) and is well established as reliable in phonological processing investigation (e.g., Katzir et al., 2005). The second task was a non-verbalizable visual-symbol matching task in which participants were presented with unfamiliar Japanese hiragana (no participants knew that they were letters from another language). Visually similar hiraganas (e.g., 女 vs. め) were presented to try to maximize difficulty (Figure 1B). This task was used to at least partially account for visual input and motor response often associated with fMRI tasks that requires processing of letters and explicit motor responses (Henson et al., 2000). Finally, the third task was a color-matching task in which participants were asked to determine whether two colors matched (Figure 1C). The pair of stimuli were of the same hue but of different lightness with close value optimized in a pilot study to avoid using names of the colors to perform the task and to maximize difficulty. Although there is no assumed relationship between color-matching and handwriting, this task was included as another task to help account for some of the confounds, such as the color dissimilarities in the stimuli used in the phonological task and the decision making nature of all three tasks. These latter two tasks were only obtained in a portion of the children $(N=18)$. We therefore performed a secondary analysis of the phonological fMRI task matched to include only those participants that also completed the visual-symbol matching and color matching control tasks when comparing between tasks. The results of the phonological fMRI task were unchanged regardless of the sample-size and were specific to the phonological task.

All three tasks utilized the same procedure. Each required participants to determine whether two visually presented stimuli matched for either the first syllables of the names of pictures, visual symbols or color. Stimuli were presented simultaneously in one condition (without enabling working memory, WM-) 


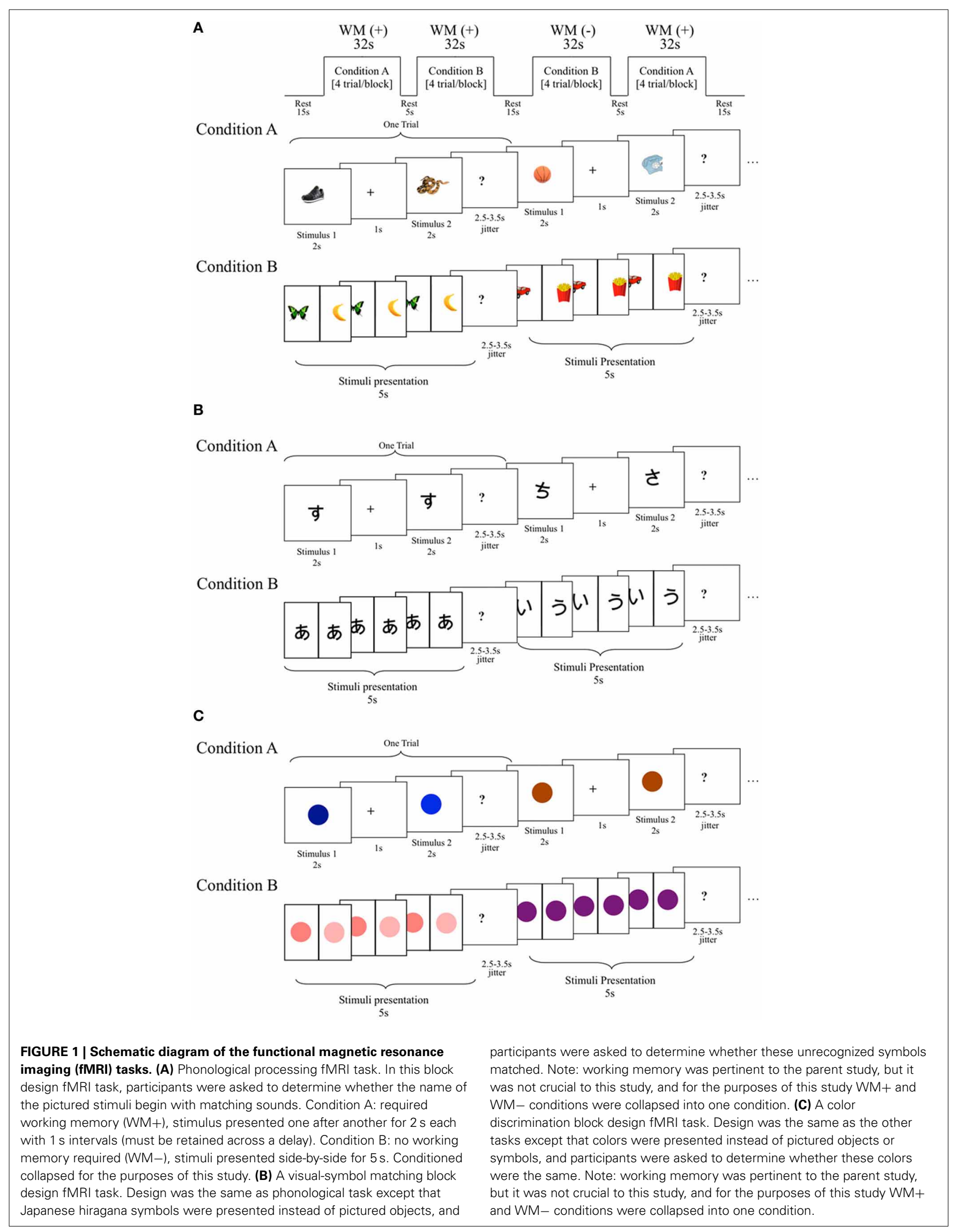


and after a small delay (enabling working memory, $\mathrm{WM}+$ ) (Figures 1A-C). In this study, we report results collapsing the two conditions. The exploration of the role of working memory in reading and writing may answer important theoretical questions and should be examined in future studies. This is not however, explored further in the current study because of the non-significant difference between conditions, which may have been due to a number of factors such as the short interstimulus interval. Rest was used as the control condition because our preliminary study showed difficulty of children performing a phonological task (sound matching of first syllable) alternating with a control condition (such as visual shape matching). We therefore opted to use the two other visual fMRI tasks to show specificity of the effects to the sound-matching task. Participants completed two runs of each task. Each of the two runs began with a $6 \mathrm{~s}$ countdown and a $2 \mathrm{~s}$ rest period. In the WM- condition, stimuli were presented side-by-side continuously for $3.5 \mathrm{~s}$ (followed by a $2.5-3.5 \mathrm{~s}$ jitter with a mean average of $3 \mathrm{~s}$ ), whereas the $\mathrm{WM}+$ condition displayed stimuli at the center one at a time for $2 \mathrm{~s}$ each with a jitter of 2.5-3.5 s (mean average $3 \mathrm{~s}$ ) between stimuli (paired stimuli were also followed by a $2.5-3.5 \mathrm{~s}$ jitter with an mean average of $3 \mathrm{~s}$ ). There were 5 trials per block. The 4 task blocks in each run were $32 \mathrm{~s}$ in duration and the order of the condition was varied from Run $1(\mathrm{WM}-\rightarrow \mathrm{WM}+\rightarrow \mathrm{WM}+\rightarrow \mathrm{WM}-$ ) and Run2 (WM+ $\rightarrow \mathrm{WM}-\rightarrow \mathrm{WM} \rightarrow \rightarrow \mathrm{WM}+)$, with a 5,15 , and $5 \mathrm{~s}$ intervals between blocks. Participants $(N=41)$ completed 2 runs, with each run being $170 \mathrm{~s}$ in length (174 s total with the first $4 \mathrm{~s}$ of the scans in each run being discarded to establish equilibrium in MR signal). All stimuli were presented against a plain, white background and participants responded with their right finger if the stimuli matched and with their left finger if they did not match. Since participants may think of different words than intended for the pictured stimuli used in the phonological task, post-hoc testing asking names of each picture was performed for each child to verify whether there were discrepancies between potentially ambiguous images that may have alternative, yet still correct, pronunciations. This was necessary to ensure accurate task performance calculation tailored for each subject. Due to the young age of participants, data were used if their task accuracy total was greater than chance. Overall accuracy as well as reaction times for all correctly answered trials are shown in Table 1.

\section{STRUCTURAL AND FUNCTIONAL MRI DATA ACQUISITION}

Imaging was conducted at the Lucas Center for Imaging at the Stanford University School of Medicine. Imaging data was acquired using GE Healthcare 3.0 Tesla 750 scanner and an 8-channel phased array head coil (GE Healthcare, Waukesha, WI). Images acquired included an axial-oblique 3D T1-weighted sequence (fast spoiled gradient recalled echo [FSPGR] pulse sequence, inversion recovery preparation pulse $[\mathrm{TI}]=400 \mathrm{~ms}$; repetition time $[\mathrm{TR}]=8.5 \mathrm{~ms}$; echo-time $[\mathrm{TE}]=3.4 \mathrm{~ms}$; flip angle $=15^{\circ}$; Receiver bandwidth $\pm 32 \mathrm{kHz}$; slice thickness $=$ $1.2 \mathrm{~mm} ; 0.86 \times 0.86 \mathrm{~mm}$ in-plane resolution; 128 slices; number of excitations $=1$; field-of-view $[\mathrm{FOV}]=22 \mathrm{~cm}$; acquisition matrix $=256 \times 192$ ). The total scan time was 4:54.

Functional MRI (fMRI) data were acquired using an axial 2D GRE Spiral In/Out (SPRLIO; Glover and Law, 2001) pulse sequence $\left(T R=2000 \mathrm{~ms} ; T E=30 \mathrm{~ms}\right.$; flip angle $=80^{\circ}$; Receiver bandwidth $+125 \mathrm{kHz}$; slice thickness $=4.0 \mathrm{~mm}$; number of slices $=31$, descending; $3.44 \times 3.44 \mathrm{~mm}$ in-plane resolution; number of temporal frames $=85 ; \mathrm{FOV}=22 \mathrm{~cm}$ ). The total duration of each task was 5:12.

\section{REGIONS OF INTERESTS (ROIs)}

Bilateral regions-of-interest (ROIs) used in this study were: (a) pars triangularis and pars opercularis of the IFG (IFGtri and IFGop, respectively) based on previous studies of language development, literacy, and handwriting in IFG (Longcamp et al., 2003, 2008), (b) Exner's region based on its role in generating graphemic-motor commands (Exner, 1881; Ritaccio et al., 1992; Roux et al., 2010; Planton et al., 2013), (c) SPL based on its involvement with complex motor sequences that contribute to the accuracy of written expression (Alexander et al., 1992; Sakurai et al., 2007), and (d) fusiform gyrus based on its role in letter (James and Gauthier, 2006) and word processing (Cohen et al., 2000). Automated Anatomical Labeling (AAL) (Tzourio-Mazoyer et al., 2002) in the WFU PickAtlas toolbox (Maldjian et al., 2003) was used to generate ROIs (a), (c), and (d). Exner's area ROI (b) was selected based on a neuroimaging study (Matsuo et al., 2003) as a region of the left precentral gyrus (PreCG, BA 6), adjacent to BA 9 and BA 44 (Talairach coordinates [TAL]: $-46,3$, 27). A sphere with a diameter of $10 \mathrm{~mm}$ centered around these coordinates was used as the Exner's area ROI.

\section{PREPROCESSING OF fMRI IMAGES}

Processing of fMRI data was performed with statistical parametric mapping software (SPM8; Wellcome Department of Cognitive Neurology, London, UK) in the MATLAB computing environment (The MathWorks, Natick, MA). After image reconstruction, each participant's data were slice time corrected, realigned to a reference volume and corrected for motion and artifacts using both SPM and in-house tools (http://www.nitrc.org/projects/art_repair/). Data were spatially normalized to Montreal Neurological Institute (MNI) space using normalization parameters obtained from the children's segmented gray matter images of high resolution T1 MRI normalized to standard template and applied to the mean functional image. Resultant images were resampled to $2 \times 2 \times 2 \mathrm{~mm}$ voxels in MNI stereotaxic space. Spatial smoothing was done with an 8-mm isotropic Gaussian kernel. Each participant's data were high pass filtered at $128 \mathrm{~s}$, and analyzed using a fixed effects model examining task; rest was not modeled and was included as implicit baseline. Five of the 46 participant's data were not included (final $N=41$ ) because of excessive motion (criteria: relative motion $<1.0 \mathrm{~mm}$ ), at or below chance task performance (criteria: accuracy $\leq 50 \%)$, and/or scanner artifact $(N=5)$.

\section{STATISTICAL ANALYSES OF FMRI DATA: MAIN ANALYSES OF INTEREST}

Statistical analysis was performed first using a fixed effects analysis for each participant modeling each condition. Task vs. rest contrasts were used for further group analysis for the purposes of this study as stated in the Functional MRI Tasks section above. Using random effects analysis, a one sample $t$-test was performed to examine brain regions that were active during the phonological 
Table 1 | Demographics and correlations.

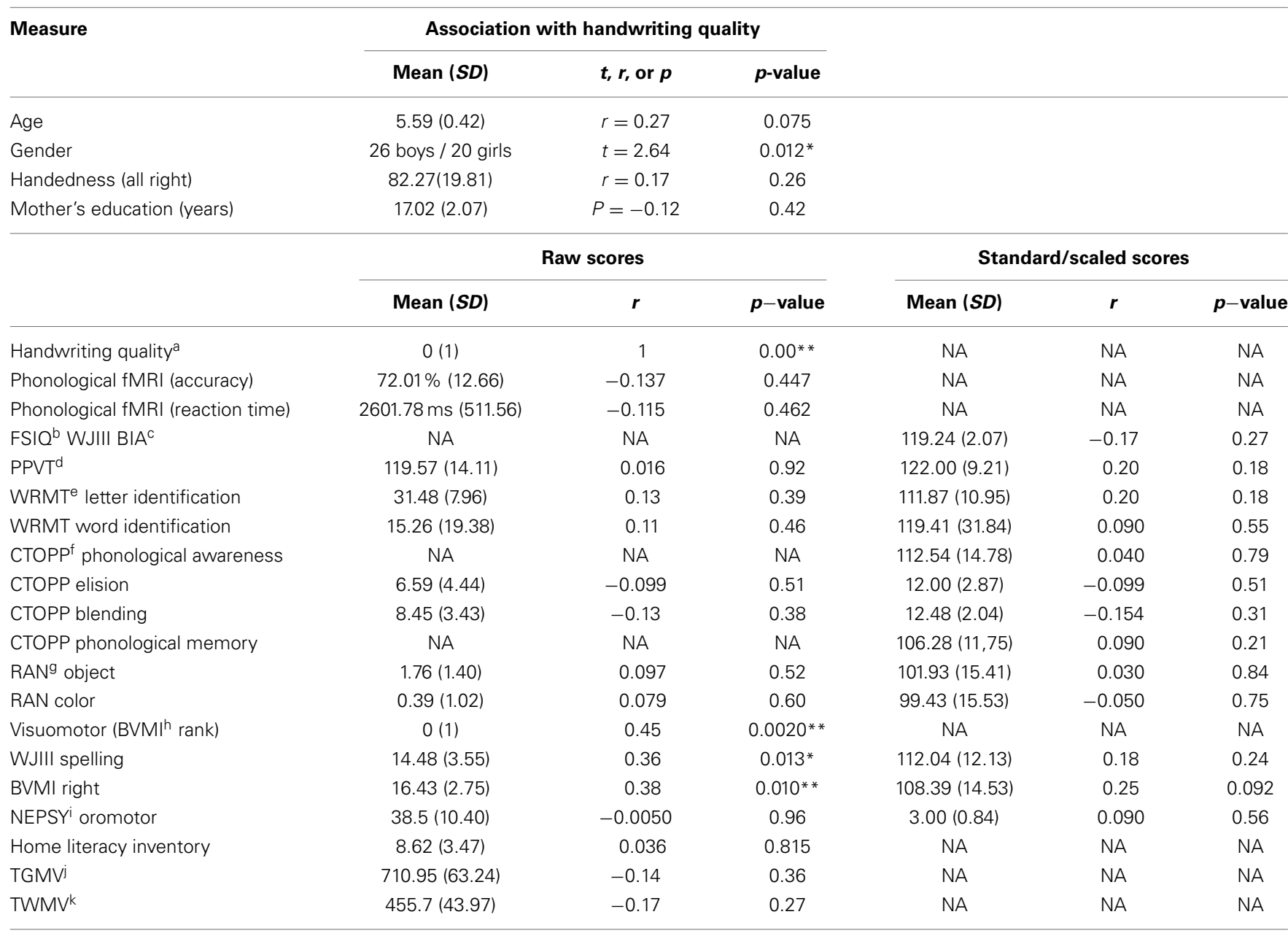

${ }^{*} p<0.05$ level (2-tailed).

${ }^{* *} p<0.01$ level (2-tailed).

${ }^{a}$ Writing samples derived from Woodcock-Johnson III Spelling (subtest from Test of Cognitive Abilities).

${ }^{b}$ Full Scale Intelligence Quotient.

${ }^{c}$ Brief Intellectual Ability.

${ }^{d}$ Peabody Picture Vocabulary Test.

e Woodcock Reading Mastery Tests.

${ }^{f}$ Comprehensive Test of Phonological Processing (Phonological Awareness = Elison+ Blending).

${ }^{g}$ Rapid Automatized Naming.

${ }^{h}$ Beery-Buktenica Developmental Test of Visual-Motor integration.

'Developmental Neuropsychological Assessment.

jTotal Gray Matter Volume.

${ }^{k}$ Total While Matter Volume.

fMRI task $[p=0.05$ family-wise error (FWE) corrected, at the whole brain level].

Next, simple correlation analysis was performed between brain activation during the fMRI tasks and handwriting skills in the ROIs using a statistical threshold of $p=0.05$ family-wise error (FWE) corrected for height using small volume correction. We also examined voxel-by-voxel associations in the whole brain at a more lenient threshold of $p=0.001$ uncorrected for height to examine whether there are any clusters outside the ROIs that showed significant effects at this more lenient threshold.

\section{STATISTICAL ANALYSES OF fMRI DATA: CONTROL ANALYSES}

Control analyses were performed in several ways. First, analyses examining associations between handwriting quality and brain activation during the phonological task regressing out the nonhandwriting motor and writing abilities such as visuomotor skills (rank order of BVMI), oromotor skills (NEPSY-II oromotor subtest), and spelling (WJ-III spelling subtest), as well as correlated demographic variables [age (there was a trend for significant effects of older age correlating with better handwriting), gender (males had significantly poorer handwriting than females)] were 
performed. Second, ROI based regression analyses between brain activation during the phonological task and these aforementioned non-handwriting motor and writing abilities were performed. Statistical threshold was set similarly to the main analysis at $p=$ 0.05 FWE corrected for the ROIs (and $p=0.001$ uncorrected for the whole brain to examine whether there are any clusters outside the ROIs that showed significant effects at this more lenient threshold). Third, whole-brain and ROI analyses were performed correlating brain activation during the supplemental visual-symbol matching and color matching tasks and handwriting skills (rank order of WJ-III spelling writing samples). Since we only had data from these tasks in half of the participants, in order to show that the significant effect in the meta-phonological task and not the supplementary tasks was not due to power issues, we went back and repeated the main correlation analysis (between brain activation during the meta-phonological processing task and handwriting skills) using a smaller sample with data from both the meta-phonological and supplementary tasks.

\section{PREPROCESSING AND STATISTICAL ANALYSIS OF T1 STRUCTURAL MR IMAGES}

Voxel-based morphometry (VBM) analysis of T1-weighted MRIs was performed using Statistical Parametric Mapping, version 8, (SPM8) (http://www.fil.ion.ucl.ac.uk/spm). After alignment to AC-PC axis, T1-weighted images were biascorrected and segmented to gray matter, white matter, and cerebrospinal fluid, using SPM8 default tissue probability maps and "New Segment" tool, which also included an affine regularization to warp images to the included International Consortium for Brain Mapping (ICBM) template, producing rigidly aligned tissue class images. Inter-subject registration was achieved with Diffeomorphic Anatomical Registration Through Exponentiated Lie Algebra (DARTEL), using default settings. Jacobian-scaled ("modulated"), warped tissue class images were created with DARTEL's "Normalize to MNI Space" tool, which spatially normalized images to MNI space, converted voxel sizes to $1.5 \times 1.5 \times 1.5 \mathrm{~mm}^{3}$ to match the DARTEL template, and smoothed images with a standard Gaussian filter of full-width at half-maximum (FWHM) equal to $8 \mathrm{~mm}$. For each participant, segmentation and normalization accuracy were manually inspected. 41 of 46 participants were included in this analysis due to usability issues caused either by artifacts or excessive motion. Statistical analyses were performed similarly to fMRI analyses using the same statistical thresholds but additionally controlling for total gray matter volume. Finally, associations between regional gray matter volume and brain activation were performed where the spatial location at least partially overlapped. The reported Talairach coordinates were converted from MNI space using the mni2tal function (http://www.mrc-cbu.cam.ac.uk/Imaging/ Common/mnispace.shtml). Talairach Daemon (Research Imaging Center, University of Texas Health Science Center; Lancaster et al., 1997, 2000) and the atlas by Talairach and Tournoux (1988) were initially used to identify Brodmann Areas. The final anatomic locations are reported according to their anatomic location overlaid on the custom template.

\section{RESULTS}

\section{BEHAVIORAL RESULTS}

Table 1 shows demographic and behavioral characteristics as well as associations between these measures and handwriting quality. Age, handedness, and maternal education, often used as a proxy for environment, did not yield any significant associations with handwriting performance (all p's $>0.05$ ). However, as one might expect based on the fact that the handwriting measure was ranked-ordered and not standardized, even though the range of ages in these children were narrow (5-6 years of age), age showed a trend for significant positive association with handwriting $\left[r_{(44)}=0.27 ; p=0.075\right]$, and gender effects were found $\left[t_{(44)}=\right.$ $2.64, p=0.012]$ with boys demonstrating significantly weaker handwriting performance as compared to girls. Further, while handwriting performance was not significantly correlated with spelling standard scores [Table 1, $r_{(44)}=0.18, p=0.24$ ], spelling raw scores were significantly related $\left[r_{(44)}=0.36, p=0.013\right]$. (Since the ranking of handwriting quality was not a standardized measure, this was expected). Visuomotor skill ratings (see above for definition) were also significantly correlated with BVMI standard scores, which is expected since visuomotor integration skill was the construct being evaluated $\left[r_{(44)}=0.658, p<0.001\right]$. We also found, as anticipated, that rater's ranking of handwriting and visuomotor skills were associated with one another $\left[r_{(44)}=0.45\right.$, $p=0.002]$.

\section{fMRI RESULTS}

First we examined brain regions that showed significant activation during the reading-related phonological processing task in all participants. We found that these emerging readers elicited significant activation at $p=0.05$ corrected in bilateral (left $>$ right) IFG, left superior, middle frontal gyrus and PreCG, left inferior parietal lobule and bilateral occipito-temporal region (Figure 2, Table 2). It is important to note that the behavioral profiles of participants included in this study are not representative of a normal population (see Table 1), so the results presented here are not yet generalizable.

Phonological activity was negatively associated with better handwriting quality in the right IFG within Broca's Area/ Brodmann Area 45 / pars triangularis [TAL: $X=44, Y=24$, $Z=15$; peak $T=3.74 ; p=0.033$ corrected; mean cluster $r_{(39)}=-0.43$; Figure 3A]. Even when performing whole-brain analysis at a lenient threshold of $p=0.001$ uncorrected, a cluster in the right IFGtri was the only region that showed a significant effect (TAL: $X=40, Y=27, Z=17$; peak $T=3.77 ; p<0.001$ uncorrected). Exner's area (TAL: $X=48$, $Y=7, Z=22$ ), although non-significant, also showed a distinctive trend in the same direction $(p=0.054$ corrected). Given Exner's well-documented involvement in handwriting, this trend was included in Figure 3). No significant positive correlations were observed either at $p=0.05$ corrected or $p=0.001$ uncorrected. Activity in the right IFGtri cluster during the phonological task was also negatively correlated with CTOPP phonological memory composite scores $(r=-0.31$, $p=0.049)$ and memory for digits subtest $(r=-0.37$, $p=0.017)$. 


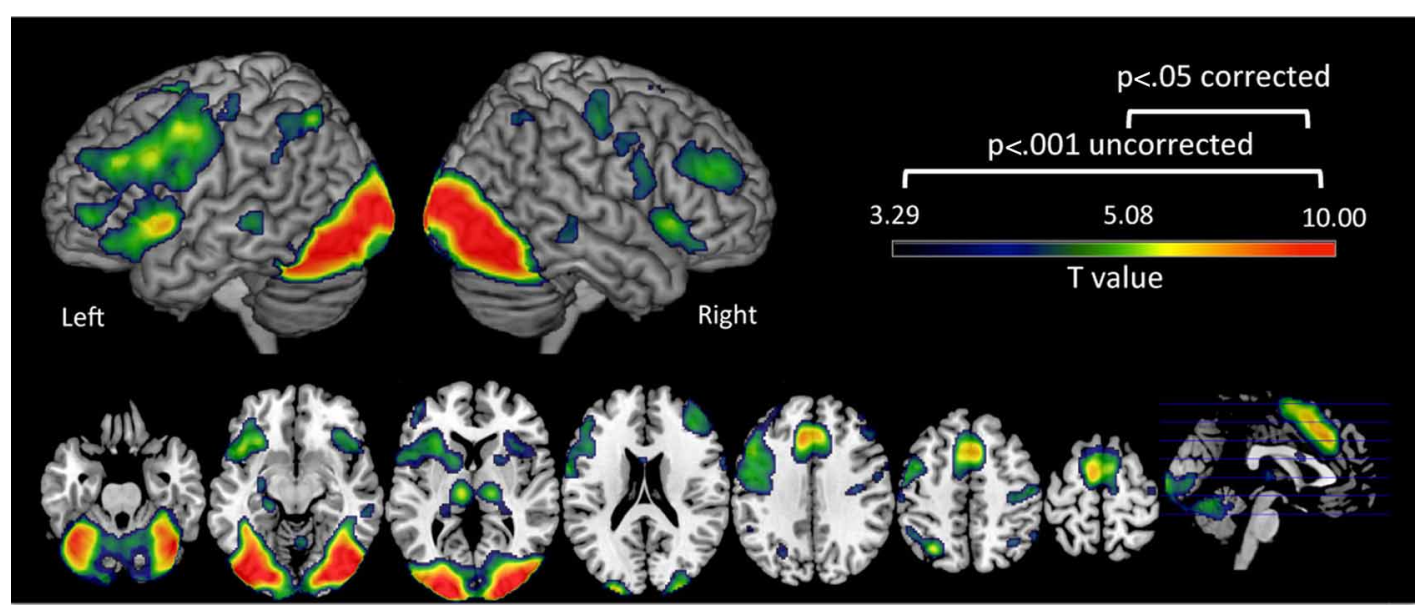

FIGURE 2 | Brain activation during the phonological processing fMRI task. Clusters in warm colors indicate those significant at $p<0.05$ family wise error (FWE) corrected. Those significant at $p<0.001$ uncorrected, cluster extent $=10$ are also included to show the extent of these clusters at a more lenient threshold. Note: Left Hemisphere is shown on left side.

Table 2 | Regional brain coordinates.

\begin{tabular}{|c|c|c|c|c|c|c|c|}
\hline \multirow[t]{2}{*}{ Brain region } & \multirow[t]{2}{*}{ Brodmann area (BA) } & \multicolumn{3}{|c|}{ TAL coordinates } & \multirow[t]{2}{*}{$T$ values (peak) } & \multirow[t]{2}{*}{$P$ value (FWE) } & \multirow[t]{2}{*}{ Cluster size (voxels) } \\
\hline & & $x$ & $y$ & $z$ & & & \\
\hline \multirow{3}{*}{$\begin{array}{l}\text { Right fusiform, inferior, middle occipital } \\
\text { gyri }\end{array}$} & 18,19 & 42 & -78 & -3 & 15.35 & $<0.001$ & 11557 \\
\hline & & 38 & -66 & -8 & 15.31 & $<0.001$ & \\
\hline & & 26 & -93 & 10 & 13.6 & $<0.001$ & \\
\hline \multirow[t]{3}{*}{ Left medial frontal, right cingulate gyri } & $9,6,32$ & -8 & 27 & 30 & 7.92 & $<0.001$ & 2125 \\
\hline & & -8 & 1 & 59 & 7.74 & $<0.001$ & \\
\hline & & 6 & 21 & 39 & 7.44 & $<0.001$ & \\
\hline \multirow{3}{*}{$\begin{array}{l}\text { Left inferior frontal, superior temporal } \\
\text { gyri, lentiform nucleus (Putamen) }\end{array}$} & 47,22 & -28 & 21 & -3 & 7.71 & $<0.001$ & 798 \\
\hline & & -46 & 11 & -4 & 7.53 & $<0.001$ & \\
\hline & & -18 & 10 & 1 & 5.43 & 0.020 & \\
\hline Left parahippocampal gyrus & 27 & -20 & -29 & -2 & 6.77 & 0.001 & 88 \\
\hline Left superior parietal lobule & 7 & -30 & -58 & 47 & 6.34 & 0.002 & 60 \\
\hline \multirow[t]{3}{*}{ Left middle frontal gyrus } & $6,9,46$ & -46 & 6 & 42 & 6.21 & 0.002 & 424 \\
\hline & & -50 & 19 & 27 & 5.96 & 0.005 & \\
\hline & & -48 & 36 & 24 & 5.94 & 0.005 & \\
\hline Left thalamus & & -10 & -17 & 5 & 6.15 & 0.003 & 114 \\
\hline Right inferior frontal gyrus & 47 & 32 & 27 & -3 & 6.13 & 0.003 & 163 \\
\hline $\begin{array}{l}\text { Right thalamus (ventral posterior lateral } \\
\text { nucleus) }\end{array}$ & & 16 & -17 & 6 & 5.67 & 0.011 & 39 \\
\hline Right thalamus & & 22 & -27 & 0 & 5.4 & 0.022 & 7 \\
\hline Right middle frontal gyrus & 10 & 40 & 40 & 18 & 5.24 & 0.033 & 8 \\
\hline Right culmen & & 4 & -65 & -10 & 5.24 & 0.034 & 8 \\
\hline Left precentral gyrus & 6 & -63 & 3 & 20 & 5.2 & 0.037 & 3 \\
\hline Right declive & & 6 & -55 & -14 & 5.19 & 0.039 & 6 \\
\hline Left inferior frontal gyrus & 10 & -44 & 47 & -2 & 5.16 & 0.041 & 2 \\
\hline Left declive & & -10 & -59 & -16 & 5.13 & 0.044 & 1 \\
\hline Right middle frontal gyrus & 10 & 44 & 48 & 20 & 5.12 & 0.046 & 2 \\
\hline Left precentral gyrus & 6 & -46 & -2 & 30 & 5.09 & 0.049 & 1 \\
\hline
\end{tabular}

Control analyses were performed in three ways. First, the negative correlation in the right IFGtri remained significant using whole-brain regression analysis of the phonological fMRI task even after regressing out variables that correlated with handwriting quality as well as other motor and writing skills such as age, gender, visuomotor skill (rank ordered BVMI responses), oromotor skills, BVMI (dominant/right hand) raw scores, and WJ-III Spelling raw scores $(r=-0.369, p=0.029)$. 


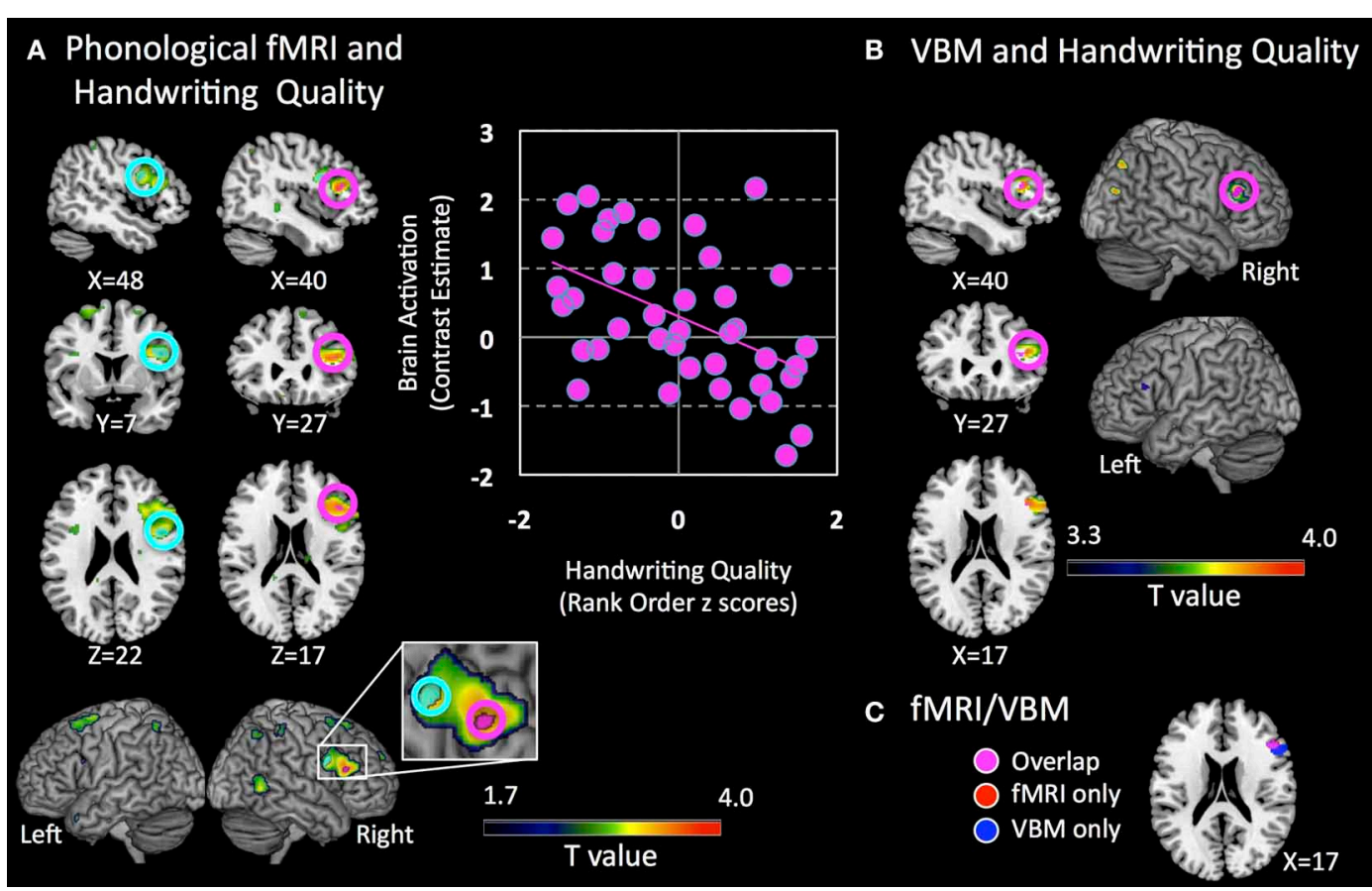

FIGURE 3 | Brain regions associated with handwriting quality.

(A) Clusters that show negative association with brain activation during a phonological processing fMRI task are shown. Pink circles indicate a cluster that is significant at $p=0.05$ corrected (right inferior frontal gyrus pars triangularis, IFGtri), and cyan circles indicate a cluster that shows a trend $p=0.054$ corrected (right Exner's area). Clusters indicate voxels significant at a lenient threshold of $p=0.05$ uncorrected to show greater extent of activation. $X Y Z$ coordinates are in Talairach coordinates. Panel on the right shows a scatter plot representation of the cluster that shows significant negative association at $p=0.05$ corrected (pink cluster). Brain activation is defined as contrast estimates, which are based on combined beta estimates of the phonological condition vs. rest. (B) Clusters that show positive association with regional gray matter volume are shown. Pink circles indicate clusters that are significant at $p=0.05$ corrected in the right IFGtri. Clusters indicate all voxels significant at $p=0.001$ uncorrected, cluster extent $=10$ as reference to show the extent of these clusters at a more lenient threshold. A small cluster in the left IFGtri is observed at this threshold. XYZ coordinates are in Talairach coordinates. (C) Voxels that show overlap in $\mathrm{fMRI}$ activation from $(\mathbf{A})$ and VBM gray matter volume from $(\mathbf{B})$ in the right inferior frontal region. Note: Left Hemisphere is shown on left side.
Second, control analyses were then performed using ROIbased (IFGtri and IFGop from AAL) and whole-brain regression between activation during phonological processing and motor and writing skills other than handwriting skills. Correlations between right IFG activation and unstandardized visuomotor skills (see Methods for definition) (peak $T=2.51 ; p=$ 0.19 corrected; $p=0.008$ uncorrected; Figure 4), oromotor skills (peak $T=3.03 ; p=0.071$ corrected; $p=0.002$ uncorrected) and spelling (peak $T=0.42 ; p=0.85$ corrected; $p=0.29$ uncorrected) were not significant, controlling for age (either by regressing age out or by using normed scores).

Third, no significant positive or negative correlation was observed with handwriting quality and brain activation during either the visual-symbol matching or color matching tasks, demonstrating that the association is likely to be specific to the phonological processing task. Since we only had data from half the sample for both the visual-symbol and color matching tasks (in what we called Cohort 1), we repeated the main correlation analysis between brain activation during phonological processing and handwriting skills using the participants included in this control analysis and still found significant results in the right IFGtri $(r=-0.49, p=0.024)$.

\section{VBM RESULTS}

We specifically examined whether there were structural correlates of the functional finding by evaluating whether there were significant associations with the right IFG regional gray matter volume and handwriting quality controlling for total gray matter volume. We found a significant positive correlation between handwriting quality and regional gray matter volume in the right IFGtri, spatially overlapping with the fMRI results (TAL: $X=40, Y=27$, $Z=17$; peak $T=3.66 ; p=0.027$ corrected; Figures $3 B, C)$. The association was however, positive and in the opposite direction to the fMRI findings. Even when the whole-brain was examined rather than the a priori hypothesized ROIs, four clusters in right IFGtri-middle frontal gyrus, left IFGtri, right middle temporal gyrus, and right postcentral gyrus - intraparietal sulcus (inferior/superior parietal lobule) were the only regions that showed a significant effect at a lenient threshold of $p=0.001$. There were no brain regions that showed significant negative association with gray matter volumes or significant positive or negative association with white matter. The positive correlation in the right IFGtri remained significant using even after regressing out variables that correlated with handwriting skills such as age, gender, visuomotor skill (rank ordered BVMI responses), BVMI (dominant/right hand) raw scores, and WJ-III Spelling raw 


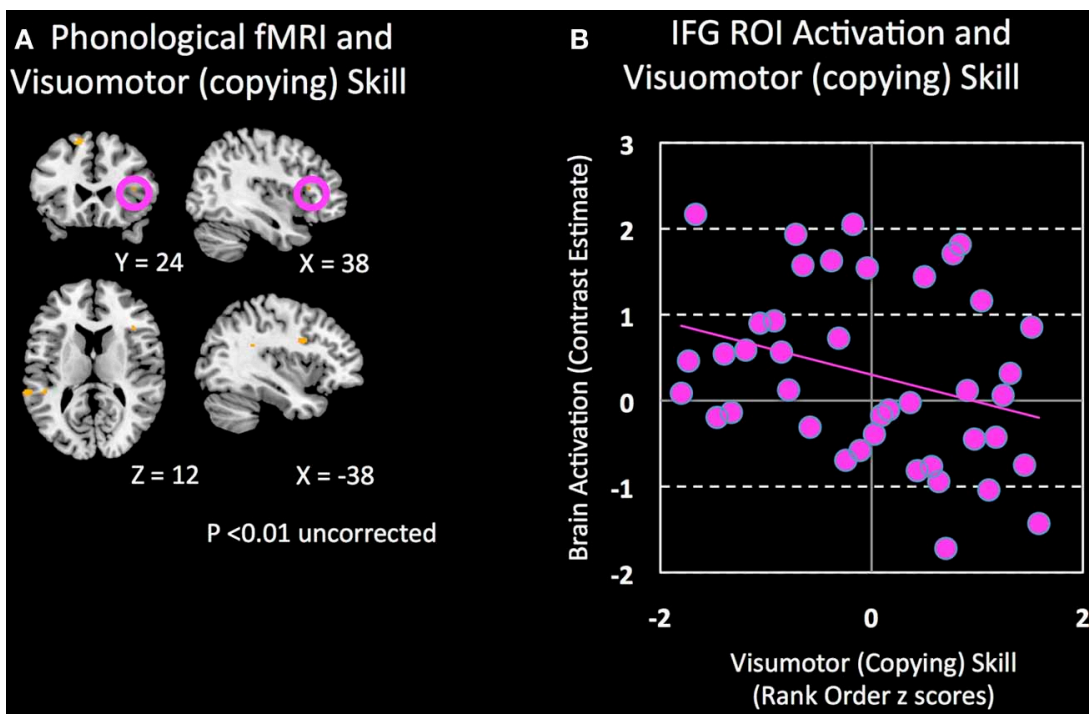

FIGURE 4 | Brain regions associated with visuomotor (copying) skill.

(A) Clusters that show a negative association ( $p=0.01$ uncorrected) between visuomotor (copying) skill and brain activation during a phonological processing $\mathrm{fMRI}$ task are shown. (B) Non-significant correlation between visuomotor (copying) skill and activation in the right IFG during phonological processing. scores $(r=0.536, p=0.001)$. Further, regional gray matter volume significantly correlated with functional activation from the main analysis $(r=-0.323, p=0.043)$.

\section{DISCUSSION}

We have presented results examining beginning writers/readers' association between handwriting quality and brain activation. Our preliminary results showed that poorer handwriting quality was associated with stronger activation of the right IFGtri when children judged whether a pair of pictures starts with the same sound. Furthermore, these results overlapped spatially with reduced regional gray matter volume in the right IFGtri in children with less proficient handwriting. Brain activation during supplementary fMRI tasks, where children judged visual similarities between pairs of unfamiliar symbols and discriminated between colors, were not associated with handwriting quality. Regional gray matter volume associations were also significantly correlated with the functional associations specific to the right IFG during the phonological task. These findings show the significance of IFG in handwriting quality in beginning writers, demonstrating that increased activation in the right IFGtri during a task likely related to the phonological processes involved in reading is associated with reduced handwriting quality, which in turn showed structural brain correlates. While our control condition was rest in our phonological fMRI task because of the young age of our participants (see Methods-Functional MRI Tasks above), we believe the task taps at least partially into phonological processing. This is because other studies using comparable tasks as well as our own study have successfully shown phonological processing related reading networks to be active during the task (see Methods). Additionally, we have included two supplementary tasks to show that the findings were at least not due to more non-specific aspects of the task such as visual perception, judgment and motoric responses. The results of this study show that the neuroanatomical properties and phonologically related neurofunctional properties of the IFG may be essential in the development of complex motor skills required in handwriting.

The IFG is a heterogeneous region with many functions. Existing literature on the IFG suggests its involvement in an exhaustive list of language abilities, including: syntactic processing (Embick et al., 2000), accessing orthographic long-term memories in the form of stored motor plans (Hillis et al., 2002; Rapp and Dufor, 2011), coordinating orthographic lexical selection and retrieval (Purcell et al., 2011), verbal working memory (Paulesu et al., 1993), letter perception and letter transcription (James and Gauthier, 2006), activation during speech generation (Liotti et al., 1994), grasping and manipulating objects (Rizzolatti et al., 1988), silent naming of manipulable objects (Grafton et al., 1997), observation of manipulable objects (Grafton et al., 1997), and when handwriting novel letterforms (Longcamp et al., 2008). Regarding its purported function in relation to writing, a recent meta-analysis of handwriting studies (Planton et al., 2013) found evidence for IFG involvement in writing, and in particular when contrasted against a control motor task (e.g., vocalization), but not for contrasts that controlled for linguistic input processing. This supports the role of the IFG in processing linguistic input during writing rather than motoric output (Planton et al., 2013). In our study, we additionally show that handwriting quality correlated not only with IFG volume, but also with activation during a task that was at least partially related to phonological processing. This suggests that at the beginning stage of reading and writing, there is a tight coupling between IFG-albeit right lateralized-and handwriting, possibly via phonological processing. It is interesting to note that handwriting quality also correlated with a behavioral measure of phonological encoding (spelling). We interpret our predominant results on the right 
hemisphere (left hemisphere involvement was present but only at subthreshold) in terms of neuronal efficiency, which we discuss below.

Although there is evidence for IFG involvement in a variety of tasks, its robust associations with phonological processing and lexical retrieval are likely the most relevant with respect to reading. Many aspects of language processing show leftward functional asymmetry in the IFG in most adults (Price, 2010). Although children show some indication of frontal left hemisphere asymmetry, the degree of asymmetry increases into adulthood (Holland et al., 2001; Szaflarski et al., 2006). Increased left functional asymmetry for language production has been linked to increased vocabulary and non-word reading scores in children (Groen et al., 2012) and more bilateral or right hemisphere IFG activations found in disabled populations, such as reading impaired dyslexics (Calvert et al., 2000; Pugh et al., 2001; Hoeft et al., 2011). Larger activation extents in the IFG have also been reported in children during linguistic tasks (Gaillard et al., 2000; Hoeft et al., 2011). This suggests a developmental reorganization and refinement of frontal language circuits through young adulthood. Our finding of a negative correlation between children's handwriting performance and right IFG activation is consistent with a common maturational process affecting handwriting and phonological processing. Children with high activation in the right IFG during phonological processing may be developmentally delayed with respect to adult-like patterns of functional asymmetry for language processing and consequently be delayed in the development of handwriting performance, either via a direct link between phonological skills and handwriting or a more general, domain independent delay. However, the specificity of our findings argues against a general delay.

An alternative account, which does not assume functional homology between the left and right IFG, is that improved handwriting is associated with increased computational efficiency or neural coding - and hence reduced BOLD signal increase-in the right IFG for reading-related functions. This phenomenon, known as neural efficiency, posits that brighter individuals use their brains more efficiently and is often used to explain the inverse relationship between brain activation and task performance (Haier et al., 1988). A recent study by Holland et al. (2011) has shown that greater recruitment of the IFG is associated with slower naming (reduced proficiency) during a picturenaming task. Further, decrease in right IFG activation during an orthographic processing task has been shown with orthographic training, a process known to contribute uniquely to handwriting, spelling, and composition (Richards et al., 2006a). Traininginduced reduction in right IFG activation has also been shown to correlate with improved phonological decoding (Richards et al., 2006b). The positive association between handwriting performance and gray matter volume may be compatible with this interpretation. Morphometric studies have found that increased regional gray matter volume may result in less energy consumption when that area is employed (Haier et al., 2004), and it is generally accepted that increased volume denotes increased cognitive capacity. This interpretation is further supported by the negative correlation between behavioral measures of phonological memory and right IFG activation during the phonological task. In our study, while both age and gender showed associations to handwriting quality (see Table 1), our findings persisted even when these factors were regressed out. Moreover, there were no significant correlations with environmental measures (e.g., Home Literacy Inventory) used as proxies to control for differential exposure to reading/writing materials. Thus, there is some indication that observed differences are not related to age or environmental differences, but instead to differences in maturational development of language related processes or neural efficiency.

Recent studies of handwriting in children have found differences in activation within the fusiform gyrus (Longcamp et al., 2008; Richards et al., 2009a,b), an area known as critical for orthographic processing and implicated both in letter and word perception, critical components for both reading development and handwriting acquisition (James and Engelhardt, 2012). Other studies note the importance of Exner's area and the SPL. Exner's area has been implicated for its role in bridging the gap between orthography and the motor programs necessary for handwriting (Roux et al., 2010; Planton et al., 2013), and the emerging consensus regarding the SPL posits that this region is involved in the abstract representation, sequential selection, and production of letter shapes (Rapp and Dufor, 2011; Planton et al., 2013; Rothlein and Rapp, 2014). We did not demonstrate a significant association between handwriting quality and neuroanatomical structure or activation in ROIs other than the IFG, such as Exner's area, fusiform gyrus and the SPL. The absence of significant results in Exner's area (though there was a trend for significance also on the right hemisphere) and the SPL may be explained by the fact that most studies that have reported these regions have used adult participants. Research has shown that in adults specific neural substrates respectively correspond to differing letter representations (Rothlein and Rapp, 2014), but this cerebral organization is likely very different in early development. It may be that the phonological processing subserved by the IFG becomes less necessary for writing as language skills become more automatic. Once this occurs, regions such as Exner's and SPL, important in the motoric and visuo-spatial component become more involved (regions thought to be specialized for fluent, automatic handwriting). It may also be the case that significant effects may have been observed in these regions if a different fMRI task was used that emphasize more motoric and visuo-spatial components, though this will not explain the lack of associations neuroanatomically. Another probable explanation is that the inverse correlation with activation in the IFG may correspond with the emergence of neural circuits in posterior writing areas in better readers. It is possible that this was not detected in our study due to the small, age-limited sample. In which case, the IFG activation may relate not to letter formation, but rather to its well-established role in motor planning and executive function. Further, while the activation observed in our study is assumed to be essential for the phonological task, some studies have shown that activation does not necessarily correspond to what is necessary for the particular tasks being administered (Rothlein and Rapp, 2014). Future studies will need to dissociate these possibilities.

The lack of association between handwriting quality and activation and neuroanatomical patterns in fusiform gyrus is more difficult to explain, especially as significant association 
with handwriting and reading (letter perception) was found in beginning writers / readers. While again, this requires further investigation (as described in limitations), it is possible that the lack of significant findings in the fusiform gyrus is related to the nature of the phonological task we used, as our task requires no orthographic processing, and hence no interaction was found with handwriting quality in the fusiform gyrus. Thus, it is very likely that if we included another fMRI task related to letter perception or orthographic processing as in James and Engelhardt (2012), we would have seen associations with handwriting in the fusiform gyrus (and SPL) also even though this will not account for the lack of neuroanatomical findings in this region.

Our study provides insights into why some children with dyslexia have been found to have poorer handwriting as well (Berninger et al., 2008). Previous literature has indicated children with dyslexia taught both word decoding and handwriting showed improvement in reading as well as orthographic decoding (Berninger et al., 2008; Berninger and Richards, 2011). It has also been shown in adults with pure alexia that reading performance can be improved through handwriting practice (Seki et al., 1995; Bartolomeo et al., 2002). Recently, a related study on the relationship between handwriting experience and neurological development in beginning readers showed that those with more experience printing and tracing activated the IFG during letter perception more than children with experience typing or copying (James and Engelhardt, 2012). Accepting past literature showing the IFG as important for linking features together to construct an organized whole, these researchers proposed that the IFG may be important for motor planning, control and execution. At a minimum, our study is distinguished from James and Engelhardt in that rather than investigating letter perception, our tasks did not include stimuli related to written languages (e.g., letters and words) and still found significant associations. Further, we find neuroanatomical evidence of associations between IFG and writing. Our findings hence provide novel findings adding to the important role of the process of writing in reading development.

\section{FUTURE DIRECTIONS AND LIMITATIONS}

Future studies investigating handwriting quality and development may assess the role of maturation, lateralization and neural efficiency related to handwriting by following children longitudinally, and by examining lower level visual and motor processing, spelling and writing compositions. Attention also plays a role in successful handwriting (McCutchen, 1996) and while we did incentivize and encourage attention, future studies may examine better ways to control or account for attention.

There are several limitations to our study that will need to be addressed in future research. First, our phonological processing task where children judged whether the initial sounds of the names of pictures matched did not have a well-matched control condition such as a picture matching condition. Although we included supplementary fMRI tasks we had available (e.g., visual matching and color matching tasks), these may have been inadequate to serve as control tasks. This determination was based on our preliminary study in young children (see MethodsFunctional MRI Tasks for details). Second, while unrealistic to keep children in kindergarten in the scanner for long periods of time, future studies may include fMRI tasks specifically related to writing, orthographic, visual and motor processing in addition to phonological processing to examine task induced differences in activation patterns as it relates to handwriting. Third, while qualitative/holistic approaches remain the most common way to assess handwriting quality (Wagner et al., 2011), there is need to find more quantitative methods, such as using computer algorithms to interpret handwriting quality and errors. Fourth, the working memory condition during fMRI was not significantly different from the non-working memory condition, and hence we were unable to address the issue of working memory in writing. Fifth, the participants included in this study were gifted compared to normative populations with standardized behavioral profiles well above average (see Table 1 ), potentially reducing the extent to which our results are generalizable. Finally, we compared a copying task (BVMI) to a spelling task (WJ-III), and there were differences in task requirements, such as encoding differences, and letters vs. symbols, as well as other potential differences such as verbal short-term memory and visual long-term memory (remembering shapes of letters); these should be dissociated in future studies. Despite these limitations, our study is an important step in identifying the neural substrates of handwriting quality in beginning writers.

\section{CONCLUSIONS}

In the current study, we provide evidence of direct neural links between handwriting quality, a skill that has been strongly associated with higher level writing skills and reading, and neural processing underlying phonological processing, which is thought to be causally related to reading acquisition. In contrast to studies focused on neurologically impaired individuals (e.g., Benson, 1979; Exner, 1881; Kaplan and Goodglass, 1981), we took a dimensional approach to investigate handwriting and have provided preliminary but novel evidence that the IFG may be a key link between phonological processing and handwriting quality during early phases of language development. The findings in the current study indicate that during early development of reading and writing skills, successful handwriting quality, measured by one's ability to shape and form letters coherently, relies on the right IFG, and that this efficiency corresponds to successful phonological processing.

\section{ACKNOWLEDGMENTS}

This study was supported by grants from the National Institute of Child Health and Human Development (K23HD054720), Child Health Research Program (CHRP), Lucile Packard Foundation for Children's Health (LPFCH), Spectrum Child Health, Clinical and Translational Science Award, and the Dyslexia Foundation (TDF)'s Extraordinary Brain Series to FH. We thank Anne Sawyer and Gary Glover for assistance with MRI data collection.

\section{REFERENCES}

Alexander, M. P., Fischer, R. S., and Friedman, R. (1992). Lesion localization in apractic agraphia. Arch. Neurol. 49, 246-251. doi: 10.1001/archneur.1992.00530270060019

Anderson, S. W., Damasio, A. R., and Damasio, H. (1990). Troubled letters but not numbers. Domain specific cognitive impairments following focal damage in frontal cortex. Brain 113(Pt. 3), 749-766. 
Bartolomeo, P., Bachoud-Lévi, A. C., Chokron, S., and Degos, J.-D. (2002). Visually- and motor-based knowledge of letters: evidence from a pure alexic patient. Neuropsychologia 40, 1363-1371. doi: 10.1016/S0028-3932(01)00209-3

Beery, K. E. (1997). The Beery-Buktenica VMI: Developmental Test of Visual-Motor Integration with Supplemental Developmental Tests of Visual Perception and Motor Coordination: Administration, Scoring, and Teaching Manual. Parsippany, NJ: Modern Curriculum Press.

Beery, K. E., and Beery, N. A. (2004). The Beery-Buktenica Developmental Test of Visual-Motor Integration with Supplemental Developmental Tests of Visual Motor Integration and Motor Coordination and Stepping Stones Age Norms From Birth to Age Six: Administration, Scoring and Teaching Manual, 5th Edn. Minneapolis, MN: NCS Pearson, Inc.

Benson, D. F. (1979). Aphasia, Alexia, and Agraphia. New York, NY: Churchill Livingstone.

Berninger, V. W., Dunn, A., Lin, S. C., and Shimada, S. (2004). School evolotion: scientist-practioner educators creating optimal learning environments for all students. J. Learn Disabil. 37, 500-508. doi: 10.1177/00222194040370060401

Berninger, V. W., Nielsen, K. H., Abbott, R. D., Wijsman, E., and Raskind, W. (2008). Writing problems in developmental dyslexia: under-recognized and under-treated. J. Sch. Psychol. 46, 1-21. doi: 10.1016/j.jsp.2006.11.008

Berninger, V. W., and Richards, T. (2011). Inter-relationships among behavioral markers, genes, brain and treatment in dyslexia and dysgraphia. Future Neurol. 5, 591-617. doi: 10.2217/fnl.10.22

Berninger, V. W., Rutberg, J. E., Abbott, R. D., Garcia, N., Anderson-Youngstrom, M., Brooks, A., et al. (2006a). Tier 1 and Tier 2 early interventions for handwriting and composing. J. Sch. Psychol. 44, 3-30. doi: 10.1016/j.jsp.2005.12.003

Berninger, V. W., and Winn, W. (2006b). "Implications of advancements in brain research and technology for writing development, writing instruction, and educational evolution," in Handbook of Writing Research, eds C. MacArthur, S. Graham, and J. Fitzgerald (New York, NY: Guilford Press), 96-114.

Byrne, B., Coventry, W. L., Olson, R. K., Hulslander, J., Wadsworth, S., DeFries, J. C., et al. (2008). A behaviour-genetic analysis of orthographic learning, spelling and decoding. J. Res. Read. 31, 8-21. doi: 10.1111/j.1467-9817.2007.00358.x

Calvert, G. A., Brammer, M. J., Morris, R. G., Williams, S. C., King, N., and Matthews, P. M. (2000). Using fMRI to study recovery from acquired dysphasia. Brain Lang. 71, 391-399. doi: 10.1006/brln.1999.2272

Castles, A., and Coltheart, M. (2004). Is there a causal link from phonological awareness to success in learning to read? Cognition 91, 77-111. doi: 10.1016/S0010-0277(03)00164-1

Cohen, L., and Dehaene, S. (2004). Specialization within the ventral stream: the case for the visual word form area. Neuroimage 22, 466-476. doi: 10.1016/j.neuroimage.2003.12.049

Cohen, L., Dehaene, S., Naccache, L., Lehéricy, S., Dehaene-Lambertz, G., Hénaff, M. A., et al. (2000). The visual word form area: spatial and temporal characterization of an initial stage of reading in normal subjects and posterior split-brain patients. Brain 123(Pt. 2), 291-307. doi: 10.1093/brain/123.2.291

Dehaene, S., Cohen, L., Sigman, M., and Vinckier, F. (2005). The neural code for written words: a proposal. Trends Cogn. Sci. 9, 335-341. doi: 10.1016/j.tics.2005.05.004

Eckert, M. A., Leonard, C. M., Richards, T. L., Aylward, E. H., Thomson, J., and Berninger, V. W. (2003). Anatomical correlates of dyslexia: frontal and cerebellar findings. Brain 126(Pt. 2), 482-494. doi: 10.1093/brain/awg026

Embick, D., Marantz, A., Miyashita, Y., O’Neil, W., and Sakai, K. L. (2000). A syntactic specialization for Broca's area. Proc. Natl. Acad. Sci. U.S.A. 97, 6150-6154. doi: 10.1073/pnas.100098897

Exner, S. (1881). Untersuchungen über die Localisation der Functionen in der Grosshirnrinde des Menschen. Vienna: Wilhelm Braumüller.

Feder, K. P., and Majnemer, A. (2007). Handwriting development, competency, and intervention. Dev. Med. Child Neurol. 49, 312-317. doi: 10.1111/j.14698749.2007.00312.x

Freeman, F. N. (1959). A new handwriting scale. Elementary Sch. J. 59, 218-221. doi: $10.1086 / 459718$

Gaillard, W. D., Hertz-Pannier, L., Mott, S. H., Barnett, A. S., LeBihan, D., and Theodore, W. H. (2000). Function anatomy of cognitive development: fMRI of verbal fluency in children and adults. Neurology 54, 180-185. doi: 10.1212/WNL.54.1.180

Glover, G. H., and Law, C. S. (2001). Spiral-in/out BOLD fMRI for increased SNR and reduced susceptibility artifacts. Magn. Reson. Med. 46, 515-522. doi: $10.1002 / \mathrm{mrm} .1222$
Goodale, M., and Milner, D. (2005). Sight Unseen. Oxford: Oxford University Press. Grafton, S. T., Fadiga, L., Arbib, M. A., and Rizzolatti, G. (1997). Premotor cortex activation during observation and naming of familiar tools. Neuroimage 6, 231-236. doi: 10.1006/nimg.1997.0293

Graham, S. (1982). Measurement of handwriting skills: a critical review. Diagnostique 8, 32-42.

Graham, S., and Weintraub, N. (1996). A review of handwriting research: progress and prospects from 1980 to 1994. Educ. Psychol. Rev. 8, 8-87. doi: 10.1007/BF01761831

Groen, M. A., Whitehouse, A. J. O., Badcock, N. A., and Bishop, D. V. M. (2012). Does cerebral lateralization develop? A study using functional transcranial Doppler ultrasound assessing lateralization for language production and visuospatial memory. Brain Behav. 2, 256-269. doi: 10.1002/ brb3.56

Haier, R. J., Jung, R. E., Yeo, R. A., Head, K., and Alkire, M. T. (2004). Structural brain variation and general intelligence. Neuroimage 23, 425-433. doi: 10.1016/j.neuroimage.2004.04.025

Haier, R. J., Siegel, K. H., Nuechterlein, E., Hazlett, J. C., Wu, J. C., Paek J., et al. (1988). Cortical glucose metabolic rate correlates of abstract reasoning and attention studied with positron emission tomography. Intelligence 12, 199-217. doi: 10.1016/0160-2896(88)90016-5

Henson, R. N. A., Burgess, N., and Frith, C. D. (2000). Recoding, storage, rehearsal and grouping in verbal short-term memory: an fMRI study. Neuropsychologia 38, 426-440. doi: 10.1016/S0028-3932(99)00098-6

Hillis, A. E., Kane, A., Tuffiash, E., Beauchamp, N. J., Barker, P. B., Jacobs, M. A., et al. (2002). Neural substrates of the cognitive processes underlying spelling: evidence from MR diffusion and perfusion imaging. Aphasiology 16, 425-438. doi: 10.1080/02687030244000248

Hoeft, F., McCandliss, B. D., Black, J. M., Gantman, A., Zakerani, N., Hulme, C., et al. (2011). Neural systems predicting long-term outcome in dyslexia. Proc. Natl. Acad. Sci. U.S.A. 108, 361-366. doi: 10.1073/pnas.1008950108

Holland, R., Leff P., Josephs, O., Galea, J. M. M., Desikan, M., Price, C., et al. (2011). Speech facilitation by left inferior frontal cortex stimulation. Curr. Biol. 21, 1403-1407. doi: 10.1016/j.cub.2011.07.021

Holland, S. K., Plante, E., Byars, A. W., Strawsburg, R. H., Schmithorst, V. J., and Ball, W. S. (2001). Normal fMRI brain activation patterns in children performing a verb generation task. Neuroimage 14, 837-843. doi: 10.1006/nimg.2001.0875

James, K. H. (2010). Sensori-motor experience leads to changes in visual processing in the developing brain. Dev. Sci. 13, 279-288. doi: 10.1111/j.14677687.2009.00883.x

James, K. H., and Engelhardt, L. (2012). The effects of handwriting experience on functional brain development in pre-literate children. Trends Neurosci. Educ. 1, 32-42. doi: 10.1016/j.tine.2012.08.001

James, K. H., and Gauthier, I. (2006). Letter processing automatically recruits a sensory-motor brain network. Neuropsychologia 44, 2937-2949. doi: 10.1016/j.neuropsychologia.2006.06.026

Kaminsky, S., and Powers, R. (1981). Remediation of handwriting difficulties, a practical approach. Acad. Ther. 17, 19-25.

Kaplan, E., and Goodglass, H. (1981). "Aphasia-related disorders," in Acquired Aphasia, 3rd Edn., ed M. T. Sarno (San Diego, CA: Academic Press), 309-333.

Karlsdottir, R., and Stefansson, T. (2002). Problems in developing functional handwriting. Percept. Mot. Skills 94, 623-662. doi: 10.2466/pms.2002.94.2.623

Katzir, T., Misra M., and Poldrack A. (2005). Imaging phonology without print: assessing the neural correlates of phonemic awareness using fMRI. Neuroimage 27, 106-115. doi: 10.1016/j.neuroimage.2005.04.013

Korkman, M., Kirk, U., and Kemp, S. I. (2007). NEPSY II, 2nd Edn. San Antonio, TX: PsychCorp/Pearson Clinical Assessment.

Lancaster, J. L., Rainey, L. H., Summerlin, J. L., Freitas, C. S., Fox, P. T., Evans, A. C., et al. (1997). Automated labeling of the human brain: a preliminary report on the development and evaluation of a forward-transform method. Hum. Brain Mapp. 5, 238-242. doi: 10.1002/(SICI)1097-0193(1997)5:4<238::AIDHBM6>3.0.CO;2-4

Lancaster, J. L., Woldorff, M. G., Parsons, L. M., Liotti, M., Freitas, C. S., Rainey, L., et al. (2000). Automated Talairach Atlas labels for functional brain mapping. Hum. Brain Mapp. 10, 120-131. doi: 10.1002/10970193(200007) 10:3<120::AID-HBM30>3.0.CO;2-8

Liotti, M., Gay, C. T., and Fox, P. T. (1994). Functional imaging and language: evidence from positron emission tomography. J. Clin. Neurophysiol. 11, 175-190. 
Longcamp, M., Anton, J.-L., Roth, M., and Velay, J.-L. (2003). Visual presentation of single letters activates a premotor area involved in writing. Neuroimage 19, 1492-1500. doi: 10.1016/S1053-8119(03)00088-0

Longcamp, M., Boucard, C., Gilhodes, J.-C., Anton, J.-L., Roth, M., Nazarian, B., et al. (2008). Learning through hand- or typewriting influences visual recognition of new graphic shapes: behavioral and functional imaging evidence. J. Cogn. Neurosci. 20, 802-815. doi: 10.1162/jocn.2008.20504

Longcamp, M., Zerbato-Poudou, M.-T., and Velay, J.-L. (2005). The influence of writing practice on letter recognition in preschool children: a comparison between handwriting and typing. Acta Psychol. 119, 67-79. doi: 10.1016/j.actpsy.2004.10.019

Lubrano, V., Roux, F.-E., and Démonet, J.-F. (2004). Writing-specific sites in frontal areas: a cortical stimulation study. J. Neurosurg. 101, 787-798. doi: 10.3171/jns.2004.101.5.0787

Maldjian, J. A., Laurienti, P. J., Kraft, R. A., and Burdette, J. H. (2003). An automated method for neuroanatomic and cytoarchitectonic atlas-based interrogation of fMRI data sets. Neuroimage 19, 1233-1239. doi: 10.1016/S10538119(03)00169-1

Marvin, C., and Ogden, N. (2002). A home literacy inventory: assessing you children's contexts for emergent literacy. Except. Young Child. 5, 2-10. doi: $10.1177 / 109625060200500201$

Matsuo, K., Kato, C., Sumiyoshi, C., Toma, K., Duy Thuy, D. H., Moriya, T., et al. (2003). Discrimination of Exner's area and the frontal eye field in humans-functional magnetic resonance imaging during language and saccade tasks. Neurosci. Lett. 340, 13-16. doi: 10.1016/S0304-3940(03) 00050-8

McCutchen, D. (1996). A capacity theory of writing: working memory in composition. Educ. Psychol. Rev. 8, 299-325. doi: 10.1007/BF01464076

Morecraft, R. J., Cipolloni, P. B., Stilwell-Morecraft, K. S., Gedney, M. T., and Pandya, D. N. (2004). Cytoarchitecture and cortical connections of the posterior cingulate and adjacent somatosensory fields in the rhesus monkey. J. Comp. Neurol. 469, 37-69. doi: 10.1002/cne.10980

O'Hare, A., and Khalid, S. (2002). The association of abnormal cerebellar function in children with developmental coordination disorder and reading difficulties. Dyslexia 8, 234-248. doi: 10.1002/dys.230

Otsuki, M., Soma, Y., Arai, T., Otsuka, A., and Tsuji, S. (1999). Pure apraxic agraphia with abnormal writing stroke sequences: report of a Japanese patient with a left superior parietal haemorrhage. J. Neurol. Neurosurg. Psychiatr. 66, 233-237. doi: 10.1136/jnnp.66.2.233

Paulesu, E., Frith, C., and Frackowiak, R. (1993). The neural correlates of the verbal component of work- ing memory. Nature 362, 342-345. doi: 10.1038/ $362342 \mathrm{a} 0$

Petrides, M., and Pandya, D. N. (1984). Projections to the frontal cortex from the posterior parietal region in the rhesus monkey. J. Comp. Neurol. 228, 105-116. doi: $10.1002 /$ cne. 902280110

Planton, S., Jucla, M., Roux F-E., and Demonet, J.-F. (2013). The "handwriting brain": a meta-analysis of neuroimaging studies of motor versus orthographic processes. Cortex 49, 2772-2787. doi: 10.1016/j.cortex.2013.05.011

Price, C. J. (2010). The anatomy of language: a review of $100 \mathrm{fMRI}$ studies published in 2009. Ann. N. Y. Acad. Sci. 1191, 62-88. doi: 10.1111/j.17496632.2010.05444.x

Pugh, K. R., Mencl, W. E., Jenner, A. R., Katz, L., Frost, S. J., Lee, J. R., et al. (2001). Neurobiological studies of reading and reading disability. J. Commun. Disord. 34, 479-492. doi: 10.1016/S0021-9924(01)00060-0

Purcell, J. J., Turkeltaub, P. E., Eden, G. F., and Rapp, B. (2011). Examining the central and peripheral processes of written word production through meta-analysis. Front. Psychol. 2:239. doi: 10.3389/fpsyg.2011.00239

Racine, M. B., Majnemer, A., Shevell, M., and Snider, L. (2008). Handwriting performance in children with attention deficit hyperactivity disorder (ADHD). J. Child Neurol. 23, 399-406. doi: 10.1177/0883073807309244

Rapp, B., and Dufor, O. (2011). The neurotopography of written word production: an $\mathrm{fMRI}$ investigation of the distribution of sensitivity to length and frequency. J. Cogn. Neurosci. 23, 4067-4081. doi: 10.1162/jocn_a_00109

Reynolds, C. R., and Kamphaus, R. W. (2006). Behavior Assessment System for Children - Second Edition (BASC-2), Self-Report of Personality Interview Edition (SRP-I). Circle Pines, MN: American Guidance Service.

Richards, T. L., Aylward, E., Berninger, V. W., Field, K., Parsons, A., Richards, A., et al. (2006a). Individual fMRI activation in orthographic mapping and morpheme mapping after orthographic or morphological spelling treatment in child dyxlexics. J. Neurolinguistics 22, 58-86. doi: 10.1016/j.jneuroling.2005.07.003

Richards, T. L., Aylward, E., Raskind, W., Abbott, R., Field, K., Parsons, A., et al. (2006b). Converging evidence for triple word form theory in dyslexia. Special Issue on Brain Imaging. Dev. Neuropsychol. 30, 547-589. doi: 10.1207/s15326942dn3001_3

Richards, T. L., Berninger, V. W., and Fayol, M. (2009a). "The writing brain of normal child writers and children with writing disabilities: generating ideas and transcribing them through the orthographic loop," in Writing: A Mosaic of Perspectives and Views, eds E. Grigorenko, E. Mambrino, and D. Preiss (New York, NY: Psychology Press), 85-105.

Richards, T. L., Berninger, V. W., and Fayol, M. (2009b). fMRI activation differences between 11-year-old good and poor spellers' access in working memory to temporary and long-term orthographic representations. J. Neurolinguistics 22, 327-353. doi: 10.1016/j.jneuroling.2008.11.002

Richards, T. L., Berninger, V. W., Stock, P., Altemeier, L., Trivedi, P., and Maravilla, K. R. (2011). Differences between good and poor child writers on fMRI contrasts for writing newly taught and highly practiced letter forms. Read. Writ. 24, 493-516. doi: 10.1007/s11145-009-9217-3

Ritaccio, A. L., Hickling, E. J., and Ramani, V. (1992). The role of dominant premotor cortex and grapheme to phoneme transformation in reading epilepsy. A neuroanatomic, neurophysiologic, and neuropsychological study. Arch. Neurol. 49, 933-939. doi: 10.1001/archneur.1992.00530330055016

Rizzolatti, G., Camarda, R., Fogassi, L., Gentilucci, M., Luppino, G., and Matelli, M. (1988). Functional organization of inferior area 6 in the macaque monkey. II. Area F5 and the control of distal movements. Exp. Brain Res. Experimentelle Hirnforschung. Expérimentation cérébrale 71, 491-507. doi: 10.1007/BF00248742

Rothlein, D., and Rapp, B. (2014). The similarity structure of distributed neural responses reveals the multiple representations of letters. NeuroImage 89, 331-344. doi: 10.1016/j.neuroimage.2013.11.054

Roux, F.-E., Draper, L., Köpke, B., and Démonet, J.-F. (2010). Who actually read Exner? Returning to the source of the frontal "writing centre" hypothesis. Cortex 46, 1204-1210. doi: 10.1016/j.cortex.2010.03.001

Roux, F.-E., Dufor, O., Giussani, C., Wamain, Y., Draper, L., Longcamp, M., et al. (2009). The graphemic/motor frontal area Exner's area revisited. Ann. Neurol. 66, 537-545. doi: 10.1002/ana.21804

Sakurai, Y., Onuma, Y., Nakazawa, G., Ugawa, Y., Momose, T., Tsuji, S., et al. (2007) Parietal dysgraphia: characterization of abnormal writing stroke sequences, character formation and character recall. Behav. Neurol. 18, 99-114. doi: $10.1155 / 2007 / 906417$

Sassoon, R. (2007). Handwriting of the Twentieth Century. Bristol: Intellect Books.

Segal, E., and Petrides, M. (2012). The anterior superior parietal lobule and its interactions with language and motor areas during writing. Eur. J. Neurosci. 35, 309-322. doi: 10.1111/j.1460-9568.2011.07937.x

Seki, K., Yajima, M., and Sugishita, M. (1995). The efficacy of kinesthetic reading treatment for pure alexia. Neuropsychologia 33, 595-609. doi: 10.1016/00283932(94)00138-F

Simner, M. L. (1998). An empirically derived basis for using geometric-form copy tasks to identify kindergarten children at risk for early school failure. Can. J. Sch. Psychol. 13, 61-76. doi: 10.1177/082957359801300206

Stewart, S. (1992). "Development of written language proficiency: methods for teaching text structure," in Communication Skills and Classroom Success, ed C. S. Simon (Eau Claire, WI: Thinking Publications), 419-432.

Szaflarski, J. P., Holland, S. K., Schmithorst, V. J., and Byars, A. W. (2006), fMRI study of language lateralization in children and adults. Hum. Brain Mapp. 27, 202-212. doi: 10.1002/hbm.20177

Talairach J., and Tournoux P. (1988). Co-planar Stereotaxic Atlas of the Human Brain. New York, NY: Thieme.

Tzourio-Mazoyer, N., Landeau, B., Papathanassiou, D., Crivello, F., Etard, O., Delcroix, N., et al. (2002). Automated anatomical labeling of activations in SPM using a macroscopic anatomical parcellation of the MNI MRI single-subject brain. Neuroimage 15, 273-289. doi: 10.1006/nimg.2001.0978

Wagner, R. K., Puranik, C. S., Foorman, B., Foster, E., Wilson, L. G., Tschinkel, E., et al. (2011). Modeling the development of written language. Read. Writ. 24, 203-220. doi: 10.1007/s11145-010-9266-7

Wagner, R. K., Torgesen, J. K., and Rashotte, C. A. (1999). Comprehensive Test of Phonological Processing (CTOPP). Austin, TX: Pro-Ed. 
Woodcock, W., Kevin, S., Mather, M., and Mather, N. (2001). Woodcock-Johnson Tests of Achievement (WJ III). Itasca, IL: Riverside Publishing.

Ziviani, J., and Elkins, J. (1984). An evaluation of handwriting performance. Educ. Rev. 36, 249-261. doi: 10.1080/0013191840 360304

Conflict of Interest Statement: The authors declare that the research was conducted in the absence of any commercial or financial relationships that could be construed as a potential conflict of interest.

Received: 19 December 2013; paper pending published: 02 February 2014; accepted: 02 March 2014; published online: 19 March 2014.
Citation: Gimenez P, Bugescu N, Black JM, Hancock R, Pugh K, Nagamine M, Kutner E, Mazaika P, Hendren R, McCandliss BD and Hoeft F (2014) Neuroimaging correlates of handwriting quality as children learn to read and write. Front. Hum. Neurosci. 8:155. doi: 10.3389/fnhum.2014.00155

This article was submitted to the journal Frontiers in Human Neuroscience.

Copyright () 2014 Gimenez, Bugescu, Black, Hancock, Pugh, Nagamine, Kutner, Mazaika, Hendren, McCandliss and Hoeft. This is an open-access article distributed under the terms of the Creative Commons Attribution License (CC BY). The use, distribution or reproduction in other forums is permitted, provided the original author(s) or licensor are credited and that the original publication in this journal is cited, in accordance with accepted academic practice. No use, distribution or reproduction is permitted which does not comply with these terms. 